\title{
Thermionic emission from defective carbon nanotubes
}

\author{
D. C. Cox, R. D. Forrest, P. R. Smith, and S. R. P. Silva ${ }^{a)}$ \\ Advanced Technology Institute, School of Electronics and Physical Science, University of Surrey, \\ Guildford GU2 7XH, United Kingdom
}

(Received 25 March 2004; accepted 9 July 2004)

\begin{abstract}
Using a nanomanipulation system contained within a scanning electron microscope we investigate the thermionic electron emission from multiwall carbon nanotubes. Peak emission currents of $65 \mathrm{nA}$ are measured. The carbon nanotubes being grown at low temperature by the chemical vapor deposition method are defective with poor thermal conductivity. We believe it is crucial for the thermal conductivity to be poor in order to obtain significant thermionic emission from the carbon nanotubes. This allows for the carbon nanotube during electron emission to be at high temperatures, and thus give higher emission efficiencies. At the highest emission current levels we estimate the temperature of the nanotubes to be approximately 2900 K. (C) 2004 American Institute of Physics.
\end{abstract}

[DOI: $10.1063 / 1.1790597]$

The superior mechanical, thermal, and electrical properties of carbon nanotubes make them candidate materials for many future technological applications. In particular, their ability to carry electrical currents three orders of magnitude higher than noble metals has lead to the suggestion that they may make excellent nanometer-scale wires and interconnects, as they avoid the electromigration problems associated with metal wires of similar dimensions. ${ }^{1}$ Indeed, the high current carrying capacity of multiwall nanotubes has been amply demonstrated in recent years ${ }^{2}$ and furthermore, studies of the failure mechanisms at these limits reported. ${ }^{3}$ In this study we investigate the high-energy transport regime in carbon nanotubes, and in particular report the observation and measurement of substantial thermionic electron emission from individual multiwall carbon nanotubes.

Studies of electrical properties of individual nanotubes are experimentally problematic, owing to the length scales involved. In order to address these problems there appears to be two distinct approaches being developed. First, nanotube dispersions can be deposited onto suitable substrates, and then using atomic force microscopy (AFM) individual nanotubes can be isolated, and placed onto conductive tracks to probe the electrical properties. ${ }^{4}$ Second, in situ manipulation of individual nanotubes can be performed in a suitably modified scanning electron microscope (SEM), where nanotubes are selected and manuevered between sharpened tungsten tips. ${ }^{5}$ While both of these approaches offer certain advantages over the other, the (SEM) manipulation method allows electrical properties to be determined during the manipulation process, and the ability to arrange nanotubes in threedimensional space, rather than the two offered by dispersions of nanotubes lying on substrates. It is for this reason that the SEM manipulation method is the one that we have developed $^{6}$ and have used to perform the measurements reported here.

Individual multiwall carbon nanotubes (MWNTs), grown by chemical vapor deposition (CVD), ${ }^{7}$ with typical diameters of $50-100 \mathrm{~nm}$, still on their substrates were selected and manipulated in situ, in real time, in a SEM, using two sharpened tungsten tips electrically connected to two

\footnotetext{
a) Author to whom correspondence should be addressed; electronic mail:
} s.silva@surrey.ac.uk voltage-current source meters, see Fig. 1. Each nanotube was removed from the substrate and connected between the two tips, where a series of $I-V$ data was collected, while still under high vacuum in the SEM (better than $2 \times 10^{-6}$ Torr). Typical $I-V$ for one of the nanotubes is shown in Fig. 2, where it can be seen that with a bias of less than $+5 \mathrm{~V}$, currents in excess of $30 \mu \mathrm{A}$ are observed, corresponding to a dissipated power of greater than $150 \mu \mathrm{W}$.

A frequent observation when the dissipated power is approximately $100 \mu \mathrm{W}$ or greater is that the secondary electron SEM signal is degraded as the voltage across the tube is raised, see Fig. 3. This continues until ultimately the image is lost and the Everhart-Thornley (ET) detector is completely flooded. However, subsequent reduction of the voltage across the nanotube restores the SEM image. Additionally, with no SEM beam, but the ET detector active, we still observe a significant signal arriving at the detector, resulting in complete flooding when high power is dissipated across the nanotube. With a nanotube acting as a wire connection between two much larger tips, clearly the only source of electrons can be from thermionic emission, resulting from the

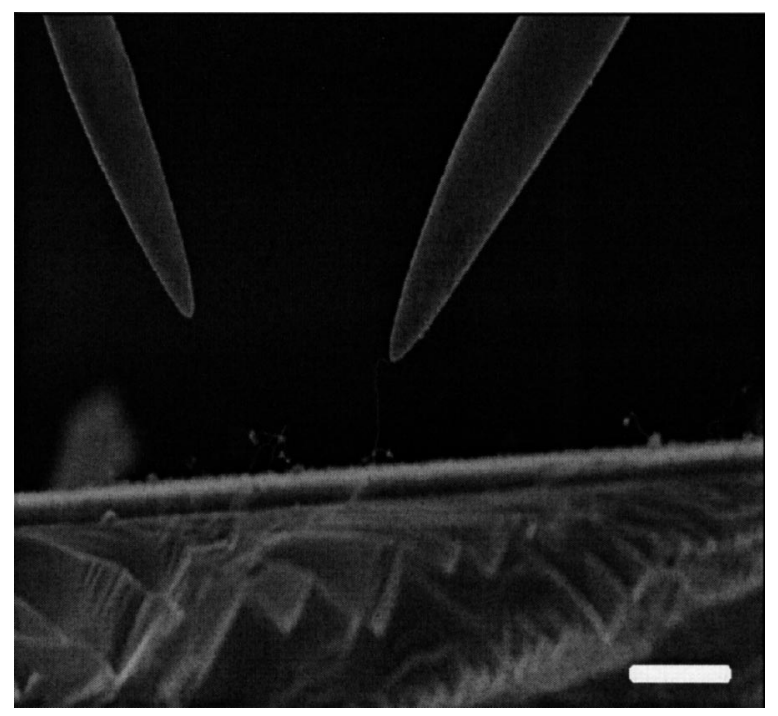

FIG. 1. SEM image of carbon nanotube being manipulated by tungsten tips in the SEM chamber. Scale bar is $10 \mu \mathrm{m}$. 


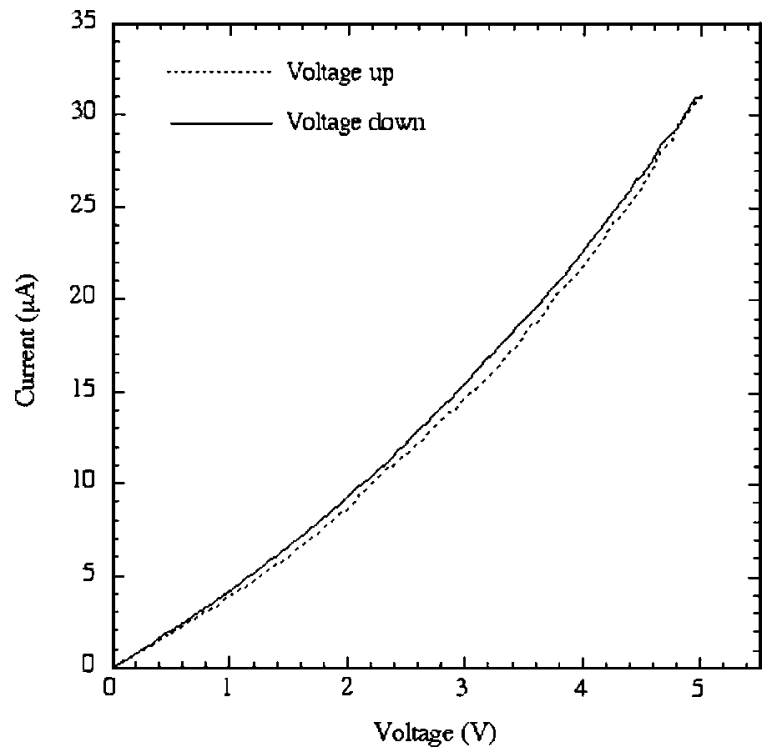

FIG. 2. Typical $I-V$ data for CVD-grown nanotube connected between the two tips.

dissipative self-heating taking place in the nanotube. To measure this a simple copper wire detector connected to a Keithley 238 current-source meter was placed in the SEM chamber near to the stage, and the tips with interconnected nanotube, were manuevered to within $10 \mathrm{~mm}$ of the wire. The copper wire was then biased to $+100 \mathrm{~V}$ as current is increased through the nanotube. As the nanotube current is allowed to rise the copper wire begins to detect thermionic emission from the tube. Typically we observe $25 \mathrm{pA}$ of thermionic emission current at around $20 \mu \mathrm{A}$ nanotube current, rising rapidly to almost $65 \mathrm{nA}$ for a rise of only $8 \mu \mathrm{A}$ carried in the tube, see Fig. 4. For the nanotubes studied here, being typically $10 \mu \mathrm{m}$ long and $100 \mathrm{~nm}$ diameter, this corresponds to an emission current density slightly over $2 \mathrm{~A} / \mathrm{cm}^{2}$. This value is regarded as the benchmark for good thermionic emitters. Using the Richardson-Dushman thermionic emission equation ${ }^{8,9}$ and making the assumption that the nanotube has only a negligible temperature gradient along the length of the tube, we estimate emission starts at $2150 \mathrm{~K}$ continuing to a peak temperature of just under $2900 \mathrm{~K}$. This value appears credible, as for significant thermionic emission to take place the nanotube must first be hot enough for thermionic emission, but at the same time lower than $3100 \mathrm{~K}$, where thermal vaporisation would occur.

Applying a simple heat transfer analysis based on dissipative self-heating, ${ }^{10}$ in a wire $10 \mu \mathrm{m}$ long, $100 \mathrm{~nm}$ diameter, dissipating $150 \mu \mathrm{W}$, provides an alternative approximation of the temperature of the nanotube. Difficulty arises with

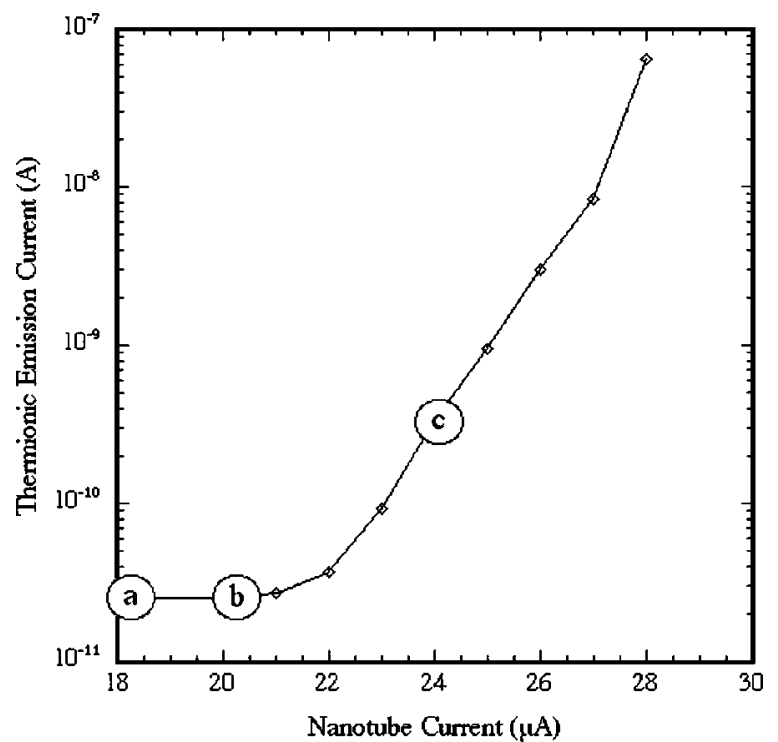

FIG. 4. Plot of thermionic emission current versus current flowing through the nanotube. The markers on the curve correspond to the images shown in Fig. 3.

this method though as an accurate thermal conductivity, $K$, is required. Experimental values vary widely from 200 (Ref. 3) to $25 \mathrm{Wm}^{-1} \mathrm{~K}^{-1}$ (Ref. 11) for MWNTs, which if applied here reveal temperatures ranging from $450-1300 \mathrm{~K}$ at peak thermionic emission. To obtain reasonable agreement between the heat transfer and thermionic emission analysis would require a thermal conductivity as low as $10 \mathrm{Wm}^{-1} \mathrm{~K}^{-1}$. The nanotubes studied here, being grown by CVD at low temperatures, would be expected to be very defective, and hence, thermal conductivity poor (as was the case in Ref. 11). Of further note is that fact that most thermal conductivity values for MWNTs are from experiments conducted at temperatures of $300 \mathrm{~K}$ or less, where in our case the thermionic emission current suggests we are in excess of $2500 \mathrm{~K}$ where it would be expected that thermal conductivity is considerably lower. It is well known that in the case of black-body radiation, the higher the temperature the more efficient the emission process. This leads to the apparent contradictory notion of a better thermionic emitter being made available with defective nanotubes, as opposed to high crystalline quality tubes. The key factor being that high-quality nanotubes with their corresponding high thermal conductivity would make poor thermionic emitters. This may be the reason why this observation of thermionic emission has not been reported previously, as most high-energy studies have concentrated on high quality nanotubes.
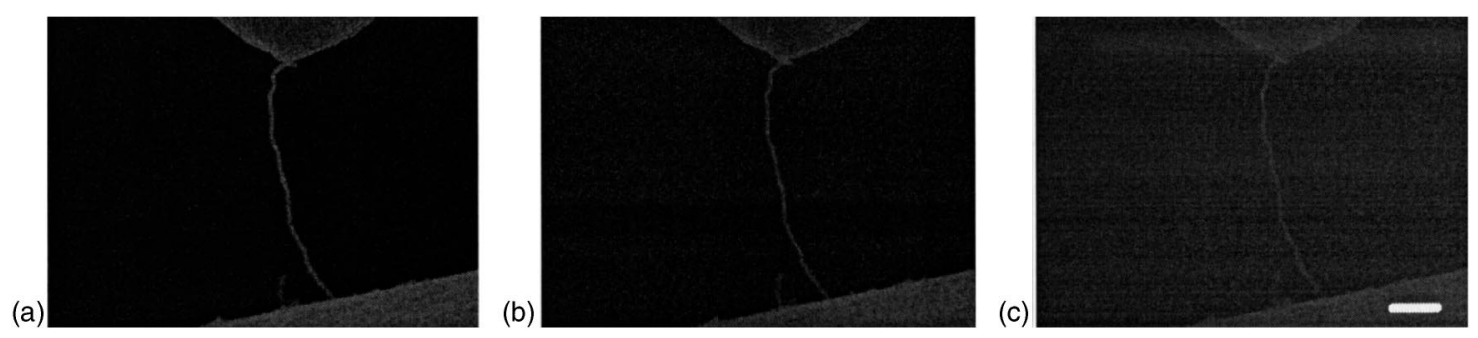

FIG. 3. Sequence of SEM images showing degrading secondary electron signal as thermionic emission from the nanotube begins to dominate. Scale bar is $2 \mu \mathrm{m}$.

Downloaded 31 Mar 2009 to 131.227.178.132. Redistribution subject to AIP license or copyright; see http://apl.aip.org/apl/copyright.jsp 
If it is assumed that defective nanotubes do indeed make good thermionic emitters it might be expected that the thermionic emission characteristics would change over time at such high temperatures, due to graphitization-like processes reducing the defect density. We have observed no significant changes in our experiments, over the duration of several minutes, and a further study is underway to investigate both the longer-term stability, and the relationship between nanotube crystalline quality and thermionic properties.

In summary, we have observed significant thermionic emission from individual MWNTs. This emission is probably made possible due to the MWNTs being grown at low temperature, and are therefore defective, resulting in a poor thermal conductivity. This raises the possibility of using similar MWNTs as miniature electron sources for $e$-beam and x-ray instruments and display applications.

The authors would like to acknowledge the EPSRC U.K. for providing funding in the form of a Portfolio Partnership Award and the Carbon Based Electronics Programme award.
${ }^{1}$ Electromigration and Electronic Device Degradation, edited by A. Christou (Wiley-Interscience, New York, 1994).

${ }^{2}$ B. Q. Wei, R. Vajtai, and P. M. Ajayan, Appl. Phys. Lett. 79, 1172 (2001). ${ }^{3}$ P. G. Collins, M. Hersam, R. Arnold, R. Martel, and Ph. Avouris, Phys. Rev. Lett. 86, 3128 (2001).

${ }^{4}$ W. Henk, Ch. Postma, M. de Jonge, Z. Yao, and C. Dekker, Phys. Rev. B 62, R10653 (2000).

${ }^{5}$ K. S. Kim, S. C. Lim, I. B. Lee, K. H. An, D. J. Bae, S. Choi, J. Yoo, and Y. H. Lee, Rev. Sci. Instrum. 74, 4021 (2003).

${ }^{6}$ D. C. Cox, R. D. Forrest, P. R. Smith, and S. R. P. Silva, NATO ASI proceedings Nanoengineered Nanofibrous Materials, 311 (Kluwer, Dordrecht, 2004).

${ }^{7}$ The nanotubes were grown by $\mathrm{CVD}$ from $\mathrm{C}_{2} \mathrm{H}_{2}$ at $550{ }^{\circ} \mathrm{C}$ on nickelcoated silicon substrates.

${ }^{8}$ Thermionic emission current density $(J)$ is given as $J=A T^{2} \exp (-\omega / k T)$, where $A$ is a constant, $T$ is temperature, $\omega$ is the electronic work function, and $k$ is the Boltzmann constant. The measurements were perfomed on a nanotube of length $10 \mu \mathrm{m}$ and $100 \mathrm{~nm}$ diam. A value of $4.95 \mathrm{eV}$ was used for the work function (from Ref. 9).

${ }^{9}$ M. Shiraishi and M. Ata, Carbon 39, 1913 (2001).

${ }^{10}$ If it assumed the MWNTs approximate to solid cylinders the peak temperature at the midpoint is $T_{p}=P_{\text {thresh }} \times$ Length $/(8 \mathrm{~K} \times$ Area $)+293$. M. Jakob, Heat Transfer (Wiley, New York, 1949).

${ }^{11}$ W. Yi, L. Lu, Z. Dian-lin, Z. W. Pan, and S. S. Xie, Phys. Rev. B 59, R9015 (1999). 